\title{
Pancreatic Mixed Adenoneuroendocrine Carcinoma
}

National Cancer Institute

\section{Source}

National Cancer Institute. Pancreatic Mixed Adenoneuroendocrine Carcinoma. NCI

Thesaurus. Code 145843.

A rare carcinoma with a poor prognosis, characterized by the presence of a mixture of exocrine and neuroendocrine malignant epithelial cells in both the pancreas and metastatic sites. Symptoms include jaundice, abdominal pain, and weight loss. 